\title{
Influenza Virus-like Particle Containing Two Different Subtypes of Hemag- glutinin Confers Protection in Mice Against Lethal Challenge With A/PR8 (H1N1) and A/HK(H3N2) Viruses
}

\author{
Farhad Rezaei ${ }^{1}$, Abbas Mirshafiey ${ }^{2}$, Shohreh Shahmahmoodi ${ }^{1}$, Zabihollah Shoja ${ }^{1}$, Nasta- \\ ran Ghavami ${ }^{1}$, Talat Mokhtari-Azad ${ }^{1, *}$ \\ ${ }^{1}$ Department of Virology, School of Public Health, Tehran University of Medical Sciences, Tehran, IR Iran \\ 2 Department of Immunology, School of Public Health, Tehran University of Medical Sciences, Tehran, IR Iran \\ * Corresponding author:Talat Mokhtari-Azad, Department of Virology, School of Public Health, Tehran University of Medical Sciences, Tehran, IR Iran. Tel.: \\ +98-2188962343, Fax: +98-2188962343, E-mail: mokhtari@tums.ac.ir.
}

\begin{abstract}
A B S T R A C T
Background: Preventing the seasonal or pandemic outbreak of influenza can be powerful and cost-effective.

objectives: In this study, we constructed a novel virus-like particle (VLP) platform that contains two hemagglutinin (HA) subtypes and evaluated immunogenicity of constructed VLP in mice.

Materials and Methods: This recombinant candidate vaccine model resulted in the expression of two HAs of H1N1 and H3N2 subtypes colocalized within a VLP. Following infection of insect cells with recombinant baculovirus co-expressing H1, H3 and M1 proteins, VLPs with size of 80-120 nm were self-assembled, budding, and released into the insect culture medium. The resulting VLPs which contained two different subtypes of hemagglutinin were purified by ultracentrifugation. The immunogenicity of VLPs was evaluated in mice following immunization. Results: Our data showed that vaccination using VLPs elicited robust levels of serum IgG, and viral neutralizing antibodies against A/PR8 (H1N1) and A/HK (H3N2) viruses. Following challenge with lethal dose of A/PR8 (H1N1) and A/HK (H3N2), vaccinated mice were protected, displaying no sign of weight loss and mortality compared to non-vaccinated control mice.
\end{abstract}

Conclusions: VLPs can serve as a promising vaccination strategy to control influenza virus.

Keywords: Orthomyxoviridae; Virus-like particles; Influenza Vaccines

Copyright (@) 2013, Iranian Red Crescent Medical Journal; Published by Kowsar Corp.

\section{Background}

Influenza is a serious respiratory disease which affects human health and is lethal. The influenza virus easily spreads as an aerosol and causes acute viral respiratory disease. Seasonal influenza epidemics solitarily account for five million severe cases worldwide (1-3). In order to prevent the seasonal or pandemic outbreak of influenza, vaccination is a cost-effective tool $(4,5)$ and protection against influenza virus is primarily derived by antibodies induced against viral hemagglutinin (HA). In addition of being a major surface glycoprotein, HA is responsible for the attachment and penetration of viral particles into the cells during the initial stages of infection $(3,6,7)$. Successful prophylactic influenza vaccines can elicit efficient antibodies against HA, which can bind to the virus and inhibit early events of the influenza infection (8). Different types of influenza vaccines are available including subunit, at-

-Article type: Research Article; Received: 08 May 2012, Revised: 27 Jul 2012, Accepted: 24 Sep 2012; DOI: 10.5812/ircmj.6252

Implication for health policy/practice/research/medical education:

The article will help the development of new strategies to produce promising vaccine against Influenza Viruses.

DPlease cite this paper as:

Rezaei F, Mirshafiey A, Shahmahmoodi Sh, Shoja Z, Ghavami N, Mokhtari-Azad T. Influenza Virus-like Particle Containing Two Different Subtypes of Hemagglutinin Confers Protection in Mice Against Lethal Challenge With A/PR8 (H1N1) and A/HK (H3N2) Viruses. Iran Red Cres Med J. 2013;15(1):75-82. DOI: 10.5812/ircmj.6252

Copyright (C) 2013, Iranian Red Crescent Medical Journal; Published by Kowsar Corp.

This is an Open Access article distributed under the terms of the Creative Commons Attribution License (http://creativecommons.org/licenses/by/3.0), which permits unrestricted use, distribution, and reproduction in any medium, provided the original work is properly cited. 
tenuated and inactivated influenza vaccines. The latter is the most widely used in the commercial scale (9-11). The major substrate for the preparation of inactivated influenza vaccines is embryonated chicken's egg (12). In currently used approaches, predominant circulating strains selected by WHO/CDC are grown in chicken eggs, chemically inactivated and semi-purified (13). Regardless of being potent immunogen, the development of these vaccines takes several months due to the identification and selection of a high yield reassorted virus strain Other drawbacks include vaccines allergic to egg proteins and difficulties in supplementing embryonated eggs particularly in case of emergency or the time when the selected influenza strain is lethal to birds $(1,14)$. In recent years several different approaches have been applied along with conventional eggbased influenza vaccine methods. Recombinant non-infectious virus-like particles (VLPs) producing in baculovirus system, structurally naïve and immunologically relevant viral antigens, seems to be a promising and novel technology for high-yielding, safe and low-cost commercial vaccines for influenza virus (1-4, 8, 11, 15-19). Several different constructs of VLPs containing either M1-HA or M1-HA-NA have been already constructed. Influenza Matrix protein (M1) plays a pivotal role in assembly and release of Virions. Additionally, contact between the cytoplasmic tails of the influenza virus membrane proteins (HA and NA) and Matrix protein is also important for formation of the budding particle $(20,21)$. Based on this phenomenon, different kinds of VLPs have been approached by different research groups (1-4, 8, 12, 15-19).

\section{Objectives}

This study was aimed to construct a VLP containing both $\mathrm{H} 1$ and $\mathrm{H} 3$ on its surface as a bivalent influenza vaccine against H1N1 and H3N2 strains.

\section{Materials and Methods}

\subsection{Cloning of H1, H3, and M1 Genes}

For the construction of the VLPs, viral RNA was extracted from A/PR8 and A/HK influenza viruses (viruses were taken as a gift from WHO, NIMR, London) using High pure Viral RNA kit (Roche Applied Science, Mannheim, Germany) followed by Reverse transcription, polymerase chain reaction (RT-PCR) with specific oligonucleotide primers and a SuperScript $₫$ III One-Step RT-PCR System with Platinum $®$ Taq High Fidelity kit (Invitrogen, USA). The produced Fragments were then visualized on $1 \%$ agarose gel at the lengths of 0.8 and $1.7 \mathrm{~KB}$ for M1 and HAs (H1 and $\mathrm{H} 3$ ), respectively. The primer pairs containing Restriction Sites for the amplification of the $\mathrm{H} 1, \mathrm{H} 3$, and $\mathrm{M} 1$ are listed as follows:

F/H1-PR38 5'- CGGGATCCATGAAGGCAAACCTACTGGTCC -3' R/H1-PR385'-GGGGTACCTCAGATGCATATTCTGCACTGCAA-3' H3/F-HK 5'-GCTCTAGAATGAAGACCATCATTGCTTTGAGC-3'

\section{H3/R-HK 5'-GGGGTACCTCAAATGCAAATGTTGCACC-3'} M1/F 5'-GGAATTCATGAGTCTTCTAACCGAGGTCG-3' M1/R 5'-GCTCTAGATCACTTGAAYCGYTGCATCTGCAC-3'

Then the products were sequenced directly using BigDye $®$ Terminator v3.1 Cycle Sequencing Kit and a 3130 Genetic Analyzer Automated Sequencer as specified by applied biosystems protocols (Foster City, CA). The nucleotide sequences were found to be identical to the sequences published in GenBank with accession numbers: EF467821, CY044261, and CY044302.

The PCR-amplified genes of H1, H3, and M1 containing restriction sites BamHI-KpnI, XbaI-KpnI, and EcoRI-XbaI, respectively, were cloned into pFastBac1 bacmid transfer vector (Invitrogen, USA) 8. Then a bacmid transfer vector expressing both HAs and M1 genes was prepared by subcloning $\mathrm{pH} 3$ and $\mathrm{pH} 1$ into $\mathrm{pM} 1 \mathrm{using}$ restriction enzymes SnaBI,HpaI, and AvrII as Pushko et al. showed (8). This resulted in a plasmid, pM1H3H1 encoding $\mathrm{M} 1, \mathrm{H} 3$, and $\mathrm{H} 1$ genes in which the expression cassette contains a polyhedrin promoter and transcription termination signals. Following transformation of E. coli DH1OBac competent cells with the constructed recombinant vector genes finally transposed into the bacmid by homologous recombination as described in the pFastBac1 protocol (Invitrogen, USA).

\subsection{Transfection of SF9 Cells by Bacmid}

The recombinant bacmid DNA was transfected into $0.8-1 \times 10^{6}$ of the Sf9 cells seeded in 6-well plates using CellFectin II reagent (Invitrogen, USA). The resulting recombinant baculovirus (rBV) collected from the culture medium on day 3 post-transfection as described by manufacturer (Invitrogen, USA) and stored at $4^{\circ} \mathrm{C}$.

\subsection{Infecting SF9 Cells With rBV Expressing H1, H3, and M1 for VLP Production}

To produce Influenza VLPs containing H1, H3, and M1 the Sf9 insect cells were seeded at a density of $2 \times 10^{7}$ per flask and infected with recombinant baculovirus (rBV) expressing the $\mathrm{H} 1, \mathrm{H} 3$, and M1 proteins at MOI (multiplicity of infection) of 3. Three days post-infection, culture supernatants containing the VLPs were harvested and clarified by low-speed centrifugation (2000 rpm for $20 \mathrm{~min}$ at $4^{\circ} \mathrm{C}$ ), followed by ultracentrifugation at $27,000 \mathrm{rpm}$ for $4 \mathrm{hrs}$ to pellet. The deposited VLPs were then suspended again in PBS at $4{ }^{\circ} \mathrm{C}$ overnight and further purified by a $20-30-60 \%$ discontinuous sucrose gradient at 27,000 rpm for 5 hrs at $4^{\circ} \mathrm{C}$.

\subsection{SDS-PAGE and Western blot Analysis}

A sodium dodecyl sulfate (SDS) $10 \%$ polyacrylamide gel and western blot analysis were used to verify the proteins content of the VLPs. 3-5ug of purified VLPs was loaded into the gel and then transferred onto a nitrocellulose membrane with the Mini Trans-Blot (Bio-Rad, CA). Following 
blotting, the membrane was blocked by $5 \%$ skimmed milk solution over night at $4{ }^{\circ} \mathrm{C}$ and then incubated with $\mathrm{H} 1, \mathrm{H} 3$, and M1 mouse IgG mAbs (1:1000 v/v for each mAb) at room temperature. Subsequently, the membrane was incubated with goat anti-mouse IgG HRP-conjugated (1:10000 v/v, Sigma) before development.

\subsection{Sandwich Capture ELISA}

To confirm whether both hemagglutinin proteins of two subtypes of influenza virus are present on the surface of the VLPs, a sandwich capture ELISA was performed. The monoclonal Abs (mAb) used for this purpose gifted from WHO, NIMR, London. The H1 mAb was coated on high sorb 96 well ELISA strips (Nunc, Denmark) overnight in $4{ }^{\circ} \mathrm{C}$ followed by blocking with skimmed mike 5\%. 20ug/ $\mathrm{ml}$ VLP was added into the wells and incubated at $37^{\circ} \mathrm{C}$ for 1 hour. After three consecutive washing with PBS containing $0.05 \%$ of Tween $20, \mathrm{H} 3 \mathrm{mAb}$ was then added and incubated for 1 hour at $37^{\circ} \mathrm{C}$. Subsequently after four consecutive washing, anti IgG mouse HRP conjugated was added and incubated at $37^{\circ} \mathrm{C}$ for 1 hour. Following final wash, TMB substrate solution was added to each well and absorbance read out at $495 \mathrm{~nm}$.

\subsection{Indirect Immunofluorescence Assay}

To confirm the expression and co-localization of recombinant H1and $\mathrm{H} 3$ proteins on the surface of sf9 cells, we performed indirect immunofluorescence assay (IIFA) using infected cells fixed with cold acetone and specific mAbs for $\mathrm{H} 1, \mathrm{H} 3$, and M1 proteins.

\subsection{Haemagglutination Assays}

For this purpose, suspensions of purified VLPs, influenza virus A/PR8 and A/HK with a protein concentration of $1 \mathrm{mg} / \mathrm{ml}$ were prepared in PBS. Two-fold Serial dilutions of 1ug of VLPs and the influenza viruses were prepared in 96-well plates and incubated at room temperature for 3060 min with $0.5 \%$ guinea pig red blood cells. The HA titer was finally inspected visually and the highest dilution capable of agglutinating red blood cells was determined.

\subsection{Haemagglutination Inhibition Assays}

Serum samples were separately treated with receptor destroying enzyme (RDE) prior to being tested with a final serum dilution of 1:10. Sera were serially diluted 2 -fold in microtiter plates. An equal volume of A/PR8 and A/HK viruses adjusted to approximately $4 \mathrm{HAU} / 25$ ul was added to each well, separately. The plates were incubated at room temperature for $60 \mathrm{~min}$ before the addition of $0.5 \%$ guinea pig erythrocytes in PBS, mixed by agitation, and left for $1 \mathrm{~h}$ at $25^{\circ} \mathrm{C}$ to allow the RBCs settle. The reciprocal of the last dilution which contained non-agglutinated RBC was considered as HAI titer. Positive and negative serum controls included in each plate. 4

\subsection{Electron Microscopy}

Coated grids of VLP sample adsorbed to the surface was stained with $1 \%$ phosphotungstic acid for 1-2 min. Excess stain wicked away with a piece of paper and then air dried for 1-3 minutes. Influenza VLPs were then visualized by using a ZEISS EM900 transmission electron microscope at $80 \mathrm{kV}$ with magnification $85 \mathrm{kX}$.

\subsection{Immunization, Challenges, and Evaluation of Humoral Immune Responses}

Female BALB/c mice (10 per group) aged 6-8 weeks were used in this study. In the first experiment, 10 female BALB/c mice per group were inoculated intranasally (i.n.) with the candidate vaccine containing a total amount of $10 \mathrm{ug}$ of VLP per dose two times on days 0 and 21. Two Negative control groups of BALB/c mice only received PBS instead of VLPs. Blood samples were taken before the primary and after booster inoculations by retro orbital plexus puncture at days 0 and 35, then sera were collected and serum antibodies were measured using HAI as well as ELISA. For second experiment on days 0 and 21,10ug of influenza VLPs were intramuscularly (i.m.) inoculated in the left hind legs of 10 female BALB/c mice per group and negative control mice (mocks) received PBS only. Before virus challenge, serum samples were collected and tested by ELISA for the presence of anti HA-specific IgG antibody. ELISA plates were coated with the inactivated influenza $\mathrm{A} / \mathrm{PR} 8$ and $\mathrm{A} / \mathrm{HK}$ viruses, followed by the addition of goat anti-mouse IgG conjugated to horseradish peroxidase (Sigma) to detect bound antibodies. Titers were considered as the highest dilution that yielded on optical density. At the end of experiments mice were injected with 100 $\mathrm{mg} / \mathrm{kg}$ intra-peritoneal ketamine and sacrificed at day 14 post-challenge.

\subsection{Statistical Analysis}

Statistical analysis of data was performed using the one way-ANOVA. P-values less than 0.05 were considered statically significant.

\section{Results}

\subsection{Expression and Characterization of Proteins}

Recombinant baculovirus expressing influenza M1, H1/ HA (A/PR/8/34, H1N1), and H3/HA (A/Hong Kong/68, H3N2) were generated by using the pFastbac bacmid transfer as mentioned above. To confirm the expressing of HAs ( $\mathrm{H} 1$ and $\mathrm{H} 3$ ) and M1 protein in the insect cell by rBV, cell lysates were subjected to SDS and Western blot analysis with monoclonal antibodies specific for HAs and M1. HA proteins ( $\mathrm{H} 1$ and $\mathrm{H} 3$ ) expressed in Sf9 cells represented a polypeptide of approximately $63 \mathrm{kDa}$ indicating an uncleaved HAO recombinant protein, as expected. The M1 
protein was also represented as a major polypeptide of approximately $29 \mathrm{kDa}$ ( Figure 1 ).

\subsection{Formation, Purification and Composition of VLPS}

The Sf9 insect cells were infected with rBV expressing both HAs ( $\mathrm{H} 1$ and $\mathrm{H} 3$ ) and M1 proteins at MOI of 3. Three days post-infection, VLPs were observed to release VLPs into the cell culture supernatant followed by purification from via a 20-30-60\% discontinuous sucrose gradient as described above. In the final preparation, western blot was used to assess the presence of $\mathrm{HAs}(\mathrm{H} 1$ and $\mathrm{H} 3)$ and M1 proteins. As indicated in Figure $1 B$, two major fractions, located in lanes 5 and 6 found to contain both M1 and HAs. Similarly, the highest haemagglutination activity (1:2048) was observed in the fractions 5 and 6 ( Figure $1 A$ ). The HA titers were 512 and 1024 per 1 ug of VLPs and homologous viruses, respectively. However, we found that the HA titer of VLPs was approximately a twofold titer lower than those of influenza virions. As indicated in previously published data, the content of HA in influenza virion is estimated to be $29 \%$ of total protein $(12,19,22,23)$. Accordingly, influenza virus contains 2.9ug HA per 10ug total protein; therefore, our generated VLPs were estimated to contain 1.8ug HA per 10ug of total VLPs protein. M1 protein was also identified in fraction 3, indicating the presence of self-assembled VLPs which contain just M1 protein with no HAs glycoproteins on the surface.

\subsection{Co-localization of Recombinant Hemaggluti- nins on Surface of SF9 Infected Cells}

To further confirm the co-localization of recombinant HAs ( $\mathrm{H} 1$ and $\mathrm{H} 3$ ) on the surface of the infected insect cells, we performed indirect immunofluorescence assay by individual $\mathrm{mAb}$ specific to each protein on a same preparation of the fixed SF9 cell co-expressing $\mathrm{H} 1, \mathrm{H} 3$, and M1 proteins. As shown in Figure 2 , the recombinant glycoproteins $\mathrm{H} 1$ and $\mathrm{H} 3$ were incorporated mostly into the cell surface while M1 proteins remained almost cytoplasmic. (Green: sf9 cells expressing proteins, Red: sf9 cells not expressing protein). We also used non-infected sf9 cell as a negative control (data not shown). In addition, randomly taken electron microscopy micrographs showed pleomorphic VLPs of different sizes of 80-120 nm range ( Figure 2D ).

\subsection{Verification of $H 1$ and $H 3$ Glycoproteins on the Surface of VLPS}

To confirm the presence of both HA protein (H1 and H3) types on the surface of VLPs, we performed a tripli

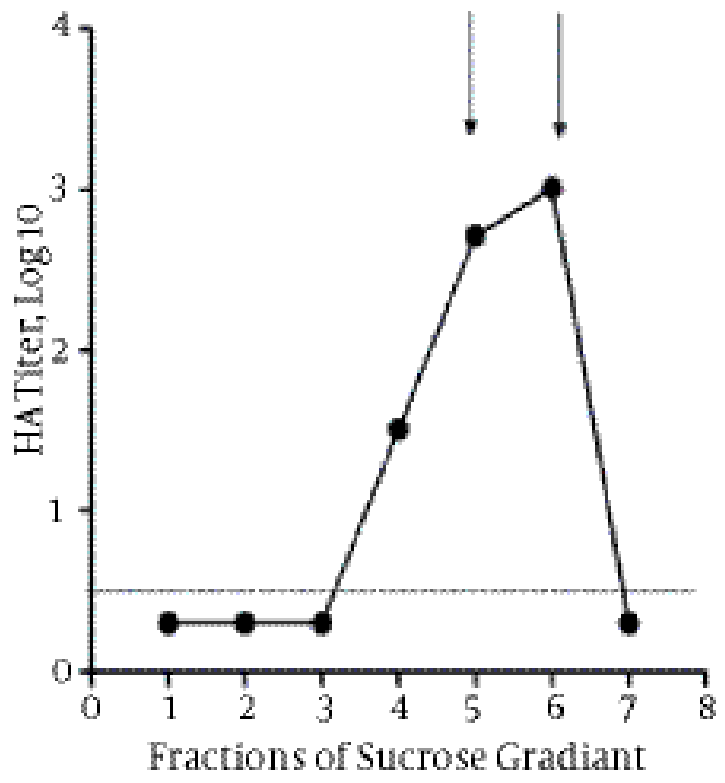

A
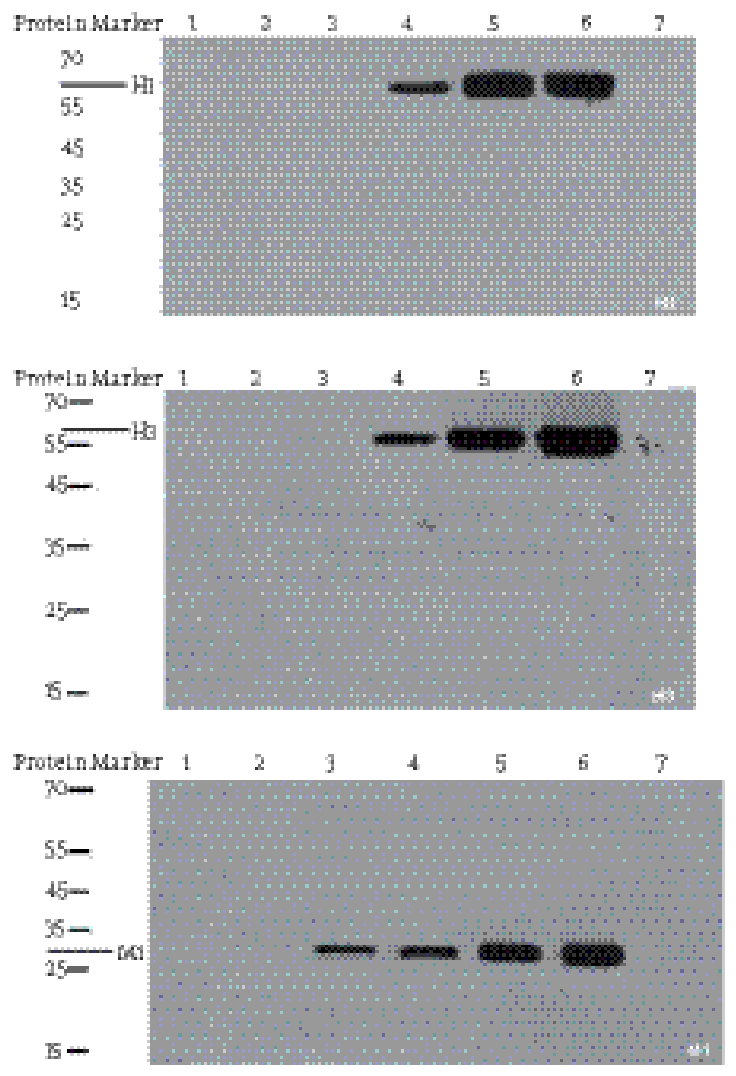

Figure 1. A) Fractions of Sucrose Gradient showing different logs of HA titer. Seven fractions were collected from sucrose gradient followed by haemagglutination assay. As indicated, the highest titers were found for fractions 5 and 6 as highlighted in arrows. Dash line indicates the base line. B) Immunobloting of different fractions was performed to determine the presence of $\mathrm{H} 1, \mathrm{H} 3$, and M1 in VLPs as illustrated 



Figure 2. Determination of hemagglutinin and matrix proteins was performed by indirect immunofluorescence of SF9 cells infected with baculoviral expressing $\mathrm{H} 1$ (A), H3 (B), and M1(C). Negative staining of electron microscopy shows the influenza VLPs comprised of the H1, H3, and M1 proteins (D). Bar represents $100 \mathrm{~nm}$

cate sandwich capture ELISA on the recovered fractions from the sucrose gradient as described in section 3.11 . Existent H1 glycoproteins on the surface of VLP were captured by $\mathrm{H} 1 \mathrm{mAb}$ coated on the wells of ELISA plate and VLPs not included the H1 protein, were excluded after washing step. In the next step, H3 mAb was added to each well and only the captured VLPs which contained the H3 glycoprotein in their context bound to the $\mathrm{H} 3 \mathrm{mAb}$. Following the addition of HRP-conjugated goat anti-mouse IgG, and chromogen substrate the absorbance was determined at $450 \mathrm{~nm}$. In addition to negative control, H1N1 and H3N2 viruses were used as antigen controls. We found the highest OD in the fraction 5 and 6 of recovered VLPs from sucrose gradients. All together, the results showed the concomitant presence of the glycoproteins on the surface of VLPs as illustrated in Figure 3.

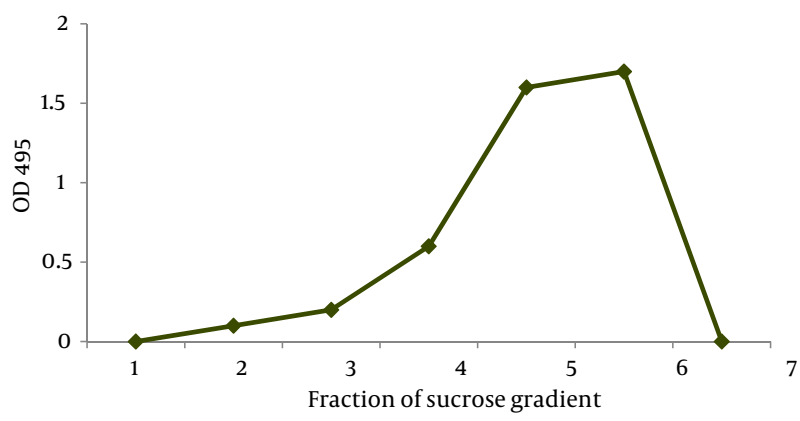

Figure 3. Concomitant presence of $\mathrm{H} 1$ and $\mathrm{H} 3$ glycoproteins on the surface of influenza VLPs. The graph shows the presence of both $\mathrm{H} 1$ and $\mathrm{H} 3$ glycoprotein types after performing a qualitative sandwich capture ELISA using specific $\mathrm{H} 1$ and $\mathrm{H} 3 \mathrm{mAb}$. Similar to the titers obtained by HA assay, the highest OD was found for fractions 5 and 6 as indicated in arrows

Based on these data, infecting insect cells with rBV expressing $\mathrm{HA}(\mathrm{H} 1$ and $\mathrm{H} 3$ ) and $\mathrm{M} 1$ proteins resulted in the expression and self-assembly of VLPs that were secreted from infected cells into Based on these data, infecting insect cells with rBV expressing $\mathrm{HA}$ (H1 and $\mathrm{H} 3$ ) and M1 proteins resulted in the expression and self-assembly of VLPs that were secreted from infected cells into the culture medium. The resulting VLPs; therefore, retained haemagglutination functions of both $\mathrm{H} 1$ and $\mathrm{H} 3$ subtypes.

\subsection{Antibody Responses, HAI and Neutralizing An- tibody}

The current study aims to evaluate the protective immune responses which were induced by bivalent influenza VLP candidate vaccine. Looking at the pre-existing antibodies to currently circulating human influenza viruses, all mice were negative (HAI $\leq 10$ ) prior to immunization. 10ug of influenza H1H3VLP was used to immunize groups of mice intranasally and intramuscularly. Following two immunizations, the H1H3VLP candidate vaccine induced HAI antibodies against the homologous strains. At week 5, all mice inoculated with the two doses (10 ug H1H3VLP per dose) had an HAI response with titers ranging from 1:80 to 1:320 against virus strains. We observed the high rise of HAI in the group of mice immunized intranasally (1:320) whereas the titer of HAI in mice immunized intramuscularly was 1:160 ( $\mathrm{P} \leq 0.05$ ). In mice that received a mock immunization we could not detect ( $<1: 10$ titer) HAI activity against any of the H1N1 and H3N2 antigens as shown in Figure $4 A$ . Additionally, the levels of IgG antibodies specific to the corresponding homologous strains A/PR8 (H1N1) and A/HK (H3N2) were determined by ELISA on plates coated with inactivated influenza viruses ( Figure $4 B$ ). The groups of mice which received bivalent influenza VLPs also showed high levels of IgG antibody specific to both viruses. Mice immunized intranasally with H1H3VLPs induced higher levels of specific IgG antibodies than those immunized intramuscularly ( $\mathrm{p} \leq 0.05$ ). Both $\mathrm{H} 1$ and $\mathrm{H} 3$ glycoproteins present in the influenza VLPs showed to be immunogenic and in terms of inducing specific immune responses against the homologous virus strains, the H1H3-VLP candidate vaccine resembles current influenza vaccines.

\subsection{Bivalent Influenza VLPs Survived Lethal Influ- enza Virus Challenges}

To determine whether immunized mice are protected against a lethal challenge, influenza H1H3-VLP immunized mice were challenged with lethal doses of A/PR8 (H1N1) and A/HK(H3N2) as illustrated in Figure 5. All H1H3 VLP-immunized mice were survived lethal virus challenges with A/PR8 (H1N1) and A/HK (H3N2) and none of them showed signs of illness or weight loss. By contrast, 3 days post-challenge mock-immunized mice controls were found to show signs of infection and illness. Concurrently these mice showed significant weight loss $(P \leq 0.05)$ and by day 6 they lost approximately $25 \%$ of their original body weight and all died by 8 days post-challenge, suggesting the applicability of H1H3-VLPs in the context of protection. 
A
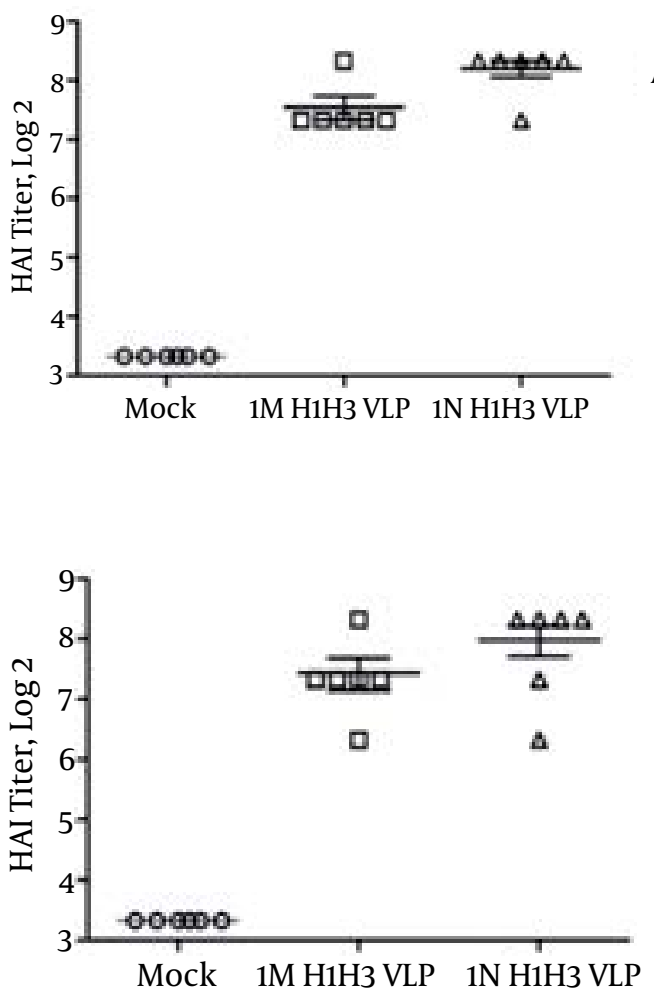

B
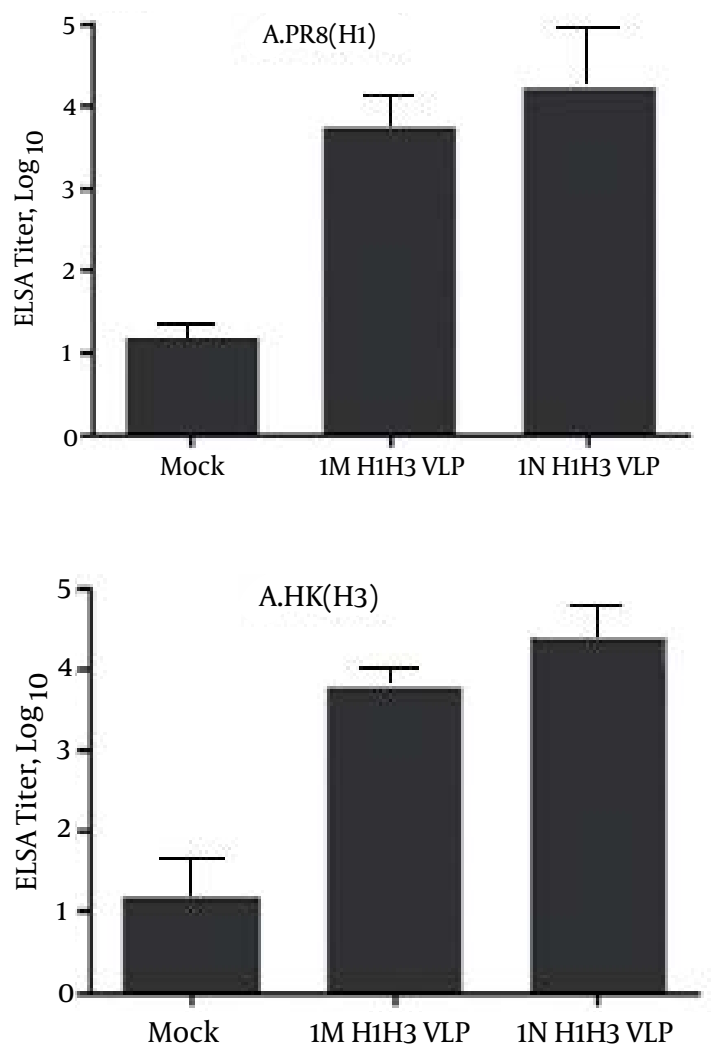

Figure 4. Protective immune responses induced by bivalent influenza VLP vaccines. Different groups of mice were immunized intranasally and intramuscularly with $10 \mathrm{ug}$ of influenza VLPs containing $\mathrm{H} 1$ and $\mathrm{H} 3$ glycoproteins. At week 5, all mice inoculated with the two doses of H1H3 VLP (10ug per dose). HAI titers and the levels of IgG antibodies specific to the corresponding homologous strains A/PR8 (H1N1) and A/HK (H3N2) are shown in Figure $4 \mathrm{~A}$ and $4 \mathrm{~B}$, respectively. (IN and IM are abbreviation for intranasally and intramuscularly vaccination routes). (Results are significant at $\mathrm{p}<0.05$, Error bars refer to mean)

Virus Challenge

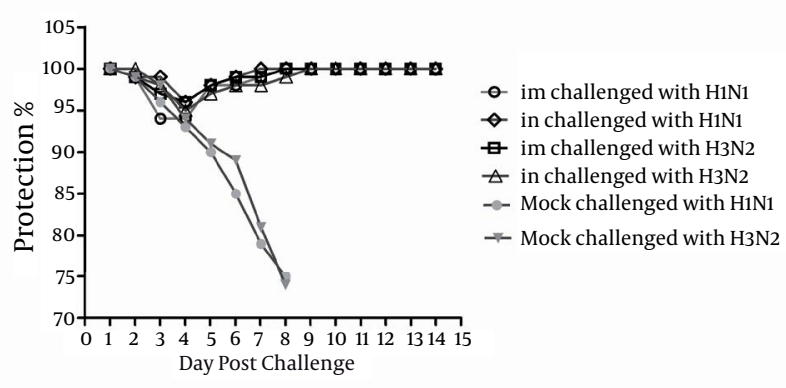

Figure 5. Protection of influenza $\mathrm{H} 1 \mathrm{H} 3 \mathrm{VLP}$-vaccinated mice following lethal virus challenges with of A/PR8 (H1N1) and A/HK(H3N2). Mice were monitored daily for two weeks to check body weight loss and percentage of survival. All VLP-immunized mice were survived lethal virus challenges with A/PR8 (H1N1) and A/HK (H3N2) and none of them showed signs of illness or weight loss. In contrast, mock-immunized controls started showing signs of illness, weight loss and morbidity at days 3 post-challenge

\section{Discussion}

Annually, emergence of new subtypes of influenza virus among human population is a great challenging issue. Lack of enough immunity of a given society to emerged subtypes is of great public health concern and yet vaccination remains a powerful and most economically public health strategy against both seasonal and pandemic influenza (10,24-26). Considering a candidate as part of influenza vaccine strategy, the development of influenza VLPs in insect cells has been suggested to overcome several drawbacks attributed to the egg-based system such as possible disruption of vaccine supplies due to the shortage of fertilized chicken embryos or potential low-yield in the case of producing highly pathogenic influenza viruses. Furthermore, the lack of viral genomic RNAs or DNAs makes VLPs as a non-infectious and safe tool for broad applications. In addition, the recombinant baculovirus expression system offers high yields of influenza VLPs and it has shown that the amount of purified VLPs via this system is approximately as the same amount of purified influenza virus ob- 
tained from egg allantoic fluid (27).

In the current study, we presented novel VLPs containing HAs from two different subtypes of influenza virus (H1 and $\mathrm{H} 3$ ). Using $\mathrm{H} 1 \mathrm{~N} 1$ and $\mathrm{H} 3 \mathrm{~N} 2$ influenza viruses, we have already shown requirements for the assembly of VLPs and their potential in influenza VLP vaccine candidate. As consistent with previously published data (1-4, $8,12,15-19)$, they demonstrates the generation of VLPs via recombinant baculovirus system expressing M1 and HAs proteins of influenza A/PR8 (H1N1) and A/HK(H3N2) viruses. The assembly of VLP are likely depend on M1 protein as its significance has been previously highlighted in both virus assembly and budding process $(8,20,21)$. Both the size and the morphology of influenza VLPs bored resembling to their corresponding virions, suggest accurate particle geometry and architecture (8). The variation seen in the size of VLPs was found to be similar to the previous observations of pleomorphic influenza virions $(1-3,8,12)$. In order to improve the coverage and the protection, the chimeric VLP candidate vaccine was developed by using two subtypes of HAs from different influenza strains. As formalin inactivated viruses may carry non-native HA proteins, the effectiveness of immune responses against these proteins can be reduced (3). Comparing to inactivated viruses, the HA glycoproteins expressed on the surface of VLPs are unmodified and native in conformation and therefore not affected by fixative. Due to the native three-dimensional conformation of expressed antigens, a chimeric influenza VLP may be more immunogenic than inactivated influenza vaccine. Our data also suggest a beneficial role of antigen sparing effects by using two proteins on the surface on influenza VLPs. However further experiments are needed to evaluate and compare the efficacy of VLP and other individual proteins as vaccines. In this study, bivalent influenza H1H3-VLPs were administered to mice intramuscularly and intranasally. Similar to the conventional influenza vaccine, the protective efficacy of the VLPs candidate vaccines depends mostly on the induction of neutralizing antibodies against the HA protein as shown here and by others (1-6, 8, 28). Accordingly, the neutralizing antibody responses were induced against the individual components of bivalent influenza VLPs. Our results indicate the bivalent influenza VLPs as an alternative influenza candidate vaccine. Influenza VLPs can also induce protective immunity comparable to the conventional influenza vaccine (9).

All together, the current study provides further evidence supporting the production of influenza VLP via insect cells as an alternative and promising approach to the egg-based conventional influenza vaccine. Additionally, this study showed that immunization with bivalent-VLPs can trigger the same type of protection induced by the current standard inactivated vaccines. The VLP approaches have the advantage by which their manufacture can overcome the necessity for embryonated chicken eggs. In the current study, we investigated the application of just two HA subtypes so far; however, in order to improve of neutralizing immunity against different subtypes, it may be possible to increase the number of HA strains included in a multivalent VLP format $(1-4,28)$. Considering the fact that influenza VLPs can be assembled from only structural proteins, it may be also possible to develop a chimeric VLP containing three or more HA glycoproteins of influenza or glycoproteins from other viruses such as parainfluenza and respiratory syncytial virus through utilizing baculoviruses or other alternative methods like DNA and virus vectors (8).

\section{Acknowledgements}

This study was part of a PhD thesis supported by Tehran University of Medical Sciences (TUMS), Grant no. 10136.

\section{Financial Disclosure}

The authors declare no conflict of interest.

\section{Funding/Support}

None declared.

\section{References}

1. Pushko P, Pearce MB, Ahmad A, Tretyakova I, Smith G, Belser JA, et al. Influenza virus-like particle can accommodate multiple subtypes of hemagglutinin and protect from multiple influenza types and subtypes. Vaccine. 2011;29(35):5911-8.

2. Pushko P, Kort T, Nathan M, Pearce MB, Smith G, Tumpey TM. Recombinant H1N1 virus-like particle vaccine elicits protective immunity in ferrets against the 2009 pandemic H1N1 influenza virus. Vaccine. 2010;28(30):4771-6.

3. Bright RA, Carter DM, Daniluk S, Toapanta FR, Ahmad A, Gavrilov V, et al. Influenza virus-like particles elicit broader immune responses than whole virion inactivated influenza virus or recombinant hemagglutinin. Vaccine. 2007;25(19):3871-8.

4. Bright RA, Carter DM, Crevar CJ, Toapanta FR, Steckbeck JD, Cole KS, et al. Cross-clade protective immune responses to influenza viruses with $\mathrm{H} 5 \mathrm{~N} 1 \mathrm{HA}$ and NA elicited by an influenza virus-like particle. PLoS One. 2008;3(1):e1501.

5. Nwe $\mathrm{N}, \mathrm{He} \mathrm{Q}$, Damrongwatanapokin S, Du Q, Manopo I, Limlamthong Y, et al. Expression of hemagglutinin protein from the avian influenza virus H5N1 in a baculovirus/insect cell system significantly enhanced by suspension culture. BMC Microbiol. 2006;6:16.

6. Crawford J, Wilkinson B, Vosnesensky A, Smith G, Garcia M, Stone $\mathrm{H}$, et al. Baculovirus-derived hemagglutinin vaccines protect against lethal influenza infections by avian $\mathrm{H} 5$ and $\mathrm{H} 7$ subtypes. Vaccine.1999;17(18):2265-74.

7. Li GX, Tian ZJ, Yu H, Jin YY, Hou SH, Zhou YJ, et al. Fusion of C3d with hemagglutinin enhances protective immunity against swine influenza virus. Res Vet Sci. 2009;86(3):406-13.

8. Pushko P, Tumpey TM, Bu F, Knell J, Robinson R, Smith G. Influenza virus-like particles comprised of the HA, NA, and M1 proteins of H9N2 influenza virus induce protective immune responses in BALB/c mice. Vaccine. 2005;23(50):5751-9.

9. Perrone LA, Ahmad A, Veguilla V, Lu X, Smith G, Katz JM, et al. Intranasal vaccination with 1918 influenza virus-like particles protects mice and ferrets from lethal 1918 and H5N1 influenza virus challenge.J Virol.2009;83(11):5726-34.

10. Robertson JS, Engelhardt OG. Developing vaccines to combat pandemic influenza. Viruses. 2010;2(2):532-46.

11. Zimmer G. RNA replicons - a new approach for influenza virus immunoprophylaxis. Viruses. 2010;2(2):413-34. 
12. Quan FS, Steinhauer D, Huang C, Ross TM, Compans RW, Kang SM. A bivalent influenza VLP vaccine confers complete inhibition of virus replication in lungs. Vaccine. 2008;26(26):3352-61.

13. Wang K, Holtz KM, Anderson K, Chubet R, Mahmoud W, Cox MM Expression and purification of an influenza hemagglutinin-one step closer to a recombinant protein-based influenza vaccine. Vaccine. 2006;24(12):2176-85.

14. Fiers W, De Filette M, Birkett A, Neirynck S, Min Jou W. A "universal" human influenza A vaccine. Virus Res. 2004;103(1-2):173-6.

15. Jin R, Lv Z, Chen Q, Quan Y, Zhang H, Li S, et al. Safety and immunogenicity of $\mathrm{H} 5 \mathrm{~N} 1$ influenza vaccine based on baculovirus surface display system of Bombyx mori. PLoS One. 2008;3(12):e3933.

16. Lee DH, Park JK, Lee YN, Song JM, Kang SM, Lee JB, et al. H9N2 avian influenza virus-like particle vaccine provides protective immunity and a strategy for the differentiation of infected from vaccinated animals. Vaccine. 2011;29(23):4003-7.

17. Mahmood K, Bright RA, Mytle N, Carter DM, Crevar CJ, Achenbach JE, et al. H5N1 VLP vaccine induced protection in ferrets against lethal challenge with highly pathogenic H5N1 influenza viruses. Vaccine. 2008;26(42):5393-9.

18. Pushko P, Tumpey TM, Van Hoeven N, Belser JA, Robinson R, Nathan $\mathrm{M}$, et al. Evaluation of influenza virus-like particles and Novasome adjuvant as candidate vaccine for avian influenza. Vaccine. 2007;25(21):4283-90.

19. Quan FS, Huang C, Compans RW, Kang SM. Virus-like particle vaccine induces protective immunity against homologous and heterologous strains of influenza virus. J Virol. 2007;81(7):3514-24.

20. Gomez-Puertas P, Mena I, Castillo M, Vivo A, Perez-Pastrana E, Por- tela A. Efficient formation of influenza virus-like particles: dependence on the expression levels of viral proteins. J Gen Virol. 1999;80 ( Pt 7):1635-45.

21. Gomez-Puertas P, Albo C, Perez-Pastrana E, Vivo A, Portela A. Influenza virus matrix protein is the major driving force in virus budding.J Virol. 2000;74(24):11538-47.

22. Epand RM, Epand RF. Thermal denaturation of influenza virus and its relationship to membrane fusion. Biochem J. 2002;365(Pt 3):841-8.

23. Tumpey TM, Renshaw M, Clements JD, Katz JM. Mucosal delivery of inactivated influenza vaccine induces B-cell-dependent heterosubtypic cross-protection against lethal influenza A H5N1 virus infection. J Virol. 2001;75(11):5141-50.

24. Bodewes R, Osterhaus AD, Rimmelzwaan GF. Targets for the induction of protective immunity against influenza a viruses. Viruses 2010;2(1):166-188.

25. Haaheim LR, Madhun AS, Cox R. Pandemic influenza vaccines - the challenges. Viruses. 2009;1(3):1089-109.

26. Kumar S, Chusid MJ, Willoughby RE, Havens PL, Kehl SC, Ledeboe NA, et al. Epidemiologic Observations from Passive and Targeted Surveillance during the First Wave of the 2009 H1N1 Influenza Pandemic in Milwaukee, WI. Viruses. 2010;2(4):782-95.

27. Wen Z, Ye L, Gao Y, Pan L, Dong K, Bu Z, et al. Immunization by in fluenza virus-like particles protects aged mice against lethal influenza virus challenge. Antiviral Res. 2009;84(3):215-24.

28. Pyo HM, Masic A, Woldeab N, Embury-Hyatt C, Lin L, Shin YK, et al Pandemic H1N1 influenza virus-like particles are immunogenic and provide protective immunity to pigs. Vaccine. 2012;30(7):1297-304. 